\title{
Thromboembolic Event Detected by Diffusion Weighted Magnetic Resonance Imaging After Coil Embolization of Cerebral Aneurysms
}

\author{
Dong-Ho Seo, Seok-Mann Yoon, Hye-Ran Park, Jai-Joon Shim, Hack-Gun Bae, II-Gyu Yun \\ Department of Neurosurgery, Soonchunhyang University Cheonan Hospital, Cheonan, Korea
}

Objective : The purpose of this study was to evaluate the occurrence rate of diffusion positive lesions (DPLs), and to assess the peri-procedural risk factors for the occurrence of DPLs in patients who underwent coil embolization of cerebral aneurysms.

Materials and Methods : A total of 304 saccular aneurysms were embolized during a seven-year period from Jan 2007 to Dec 2013. Of these, postoperative diffusion-weighted images were obtained in 186 procedures. There were 100 ruptured aneurysm and 86 unruptured aneurysms. The coiling procedures were as follows: simple coiling in 96, balloon assisted coiling (BAC) in 39, and stent assisted coiling (SAC) in 51 aneurysms. Clinical, angiographic and procedural factors were analyzed in relation to the occurrence of DPLs.

Results : Overall, DPLs were observed in 50.5\%. In unruptured aneurysms, DPLs occurred in $23.5 \%$ of BAC, $41.9 \%$ of SAC and $57.7 \%$ of simple coiling $(\rho=0.08)$. Among ruptured aneurysms, DPLs occurred in $63.6 \%$ of BAC, $62.5 \%$ of SAC and $54.3 \%$ of simple coiling $(p=0.71)$. DPLs had a tendency to increase in ruptured aneurysms compared with unruptured aneurysms (57\% vs. 43\%, $p=0.077$ ). Logistic regression analysis revealed that age $>55$ years was the only independent risk factor for the occurrence of DPLs.

Conclusion : DPLs occured more frequently in ruptured aneurysm and at an older age. Although most DPLs are asymptomatic, careful manipulation of cerebral or extracerebral arteries using various endovascular devices is important to reducing the occurrence of DPLs. BAC appeared to reduce occurrence of TE events in patient with unruptured aneurysm.

Keywords Thromboembolism, Aneurysm, Embolization, Balloon assisted coiling, Diffusion positive lesions
J Cerebrovasc Endovasc Neurosurg.

2014 September; 16(3):175-183

Received : 15 May 2014

Revised : 14 July 2014

Accepted : 24 July 2014

Correspondence to Seok-Mann Yoon Department of Neurosurgery, Soonchunhyang University Cheonan Hospital 23-20 Bongmyeong -dong, Cheonan 330-721, Korea

Tel : 82-41-570-3649

Fax : 82-41-572-9297

E-mail : smyoon@sch.ac.kr

ORCID : http://orcid.org/0000-0002-0048-9309
This is an Open Access article distributed under the terms of the Creative Commons Attribution NonCommercial License /http://creativecommons.org/licenses/by-nc/3.0) which permits unrestricted noncommercial use, distribution, and reproduction in any medium, provided the original work is properly cited.

\section{INTRODUCTION}

Thromboembolic (TE) complication is the most common complication of endovascular embolization of cerebral aneurysms. ${ }^{1323) 24) 27)}$ Although the incidence of diffusion positive lesions (DPLs) has a reported range of $10-61 \%$ following coil embolization of cerebral aneurysms, 2)4)12-14)17)19)20)23-25)27) most high signal intensity lesions on diffusion-weighted image (DWI) after embolization were asymptomatic. DPLs can be caused by various intra-procedural embolic sources. Endovascular complications can occur more frequently if the proce- 


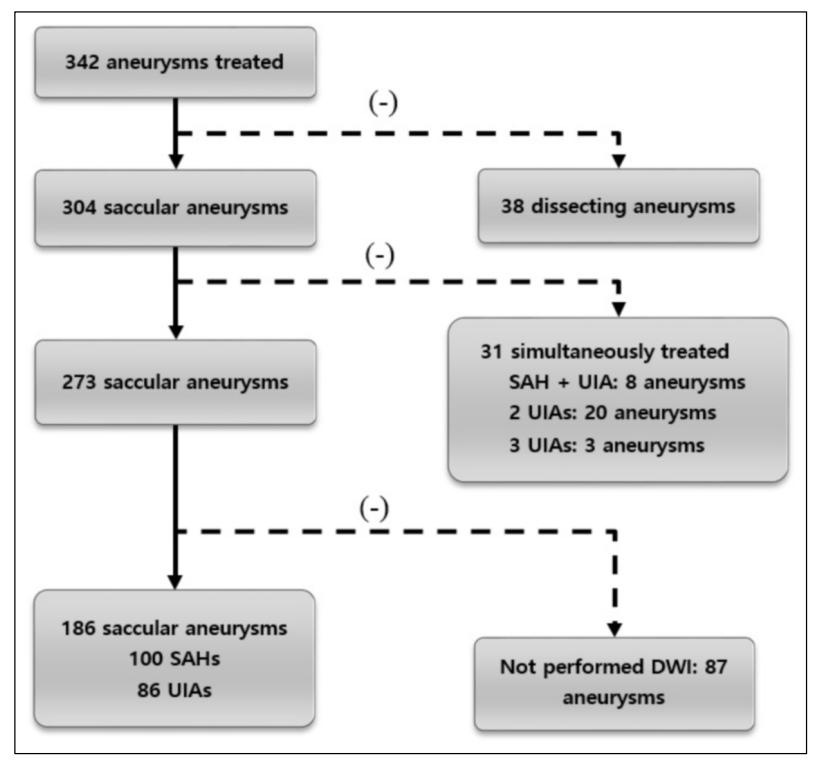

Fig. 1. Flow chart showing the patients who were selected in this study. $\mathrm{SAH}=$ subarachnoid hemorrhage; UIA = unruptured aneurysm; $D W I$ = diffusion-weighted image

dure is more complicated. The use of many devices in the guiding catheter increases the risk of complications. Therefore, balloon or stent assisted coiling (SAC) may increase TE, compared with the simple microcatheter technique. ${ }^{1114) 24)}$ However, there have been conflicting results on the effect of balloon assisted coiling (BAC) on the occurrence of TE events. ${ }^{\left.\left.1)^{6}\right)(12) 18192121\right)}$ The purpose of this study was to evaluate the incidence and risk factors for DPLs, particularly the role of BAC on the occurrence of DPLs.

\section{MATERIALS AND METHODS}

A total of 342 aneurysms were embolized in 280 patients during a seven-year period from January 2007 to Dec 2013. Of these, the following aneurysms were excluded: 38 dissecting aneurysms and 31 simultaneously treated multiple aneurysms. Multiple aneurysms treated in different sessions were included. Of the remaining 273 saccular aneurysms, postoperative DWI was not obtained in 87 aneurysms within 24 hours because of poor clinical condition, or applied mechanical ventilator status. Thus, this study in- cluded 186 procedures performed in patients who underwent postoperative DWI within 24 hours (Fig. 1). There were 100 ruptured and 86 unruptured aneurysms. The coiling procedures were as follows: simple coiling in 96, BAC in 39, and SAC in 51 aneurysms. Clinical, angiographic and procedure related events were analyzed in relation to the occurrence of DPLs in patients. All data were reviewed retrospectively using the aneurysm embolization database, picture archiving and communication system (PACS) imaging and inpatient clinical charts.

\section{Endovascular procedures}

All procedures were performed under general anesthesia by a single neurosurgeon. Patients who were expected to require stent or balloon assistance in unruptured aneurysms received dual antiplatelet therapy. Aspirin and clopidogrel were administered at doses of $100 \mathrm{mg}$ and $75 \mathrm{mg}$, respectively, starting at least five days before the procedure in unruptured aneurysm. However, in ruptured aneurysms, loading doses of $300-600 \mathrm{mg}$ of clopidogrel and $300 \mathrm{mg}$ of aspirin were given per L-tube in patients treated with stent assisted coiling.

Baseline activated clotting time (ACT) measurement was performed after placement of the femoral sheath. Intra-procedurally, 3000 units of heparin was typically injected intravenously by the anesthesia team nurse, in all patients. Target ACT during the procedure was at least more than twice the baseline $(250-300 \mathrm{sec})$ in all patients. The timing of heparin injection was different, depending on the rupture status. Heparin was injected either after placement of the framing coil placement, or just before balloon inflation in BAC in ruptured aneurysms. In unruptured aneurysms, heparin was injected after placement of the guiding catheter. In most cases, we preferred the microcatheter jailing technique in performance of the SAC procedure. Balloon assisted technique consisted of repeated inflation and deflation of the balloon before detachment of coils in order to verify stability of the coil mass as described by Morret. ${ }^{12)}$ The decision to 
Table 1. Summary of clinical characteristics according to ruptured and unruptured aneurysms in 186 patients

\begin{tabular}{|c|c|c|c|c|}
\hline Characteristic & Ruptured group & Unruptured group & Total & $\rho$ value \\
\hline Number of patient & 100 & 86 & 186 & \\
\hline Age & $54.6 \pm 13.1$ & $59.7 \pm 12.9$ & $56.9 \pm 13.2$ & 0.008 \\
\hline Female sex (\%) & $65(65.0)$ & $71(82.6)$ & $136(73.1)$ & 0.008 \\
\hline HHG (\%) & & & & 0.000 \\
\hline 0 & - & $86(100.0)$ & $86(46.2)$ & \\
\hline 1 & $5(5.0)$ & - & $5(2.7)$ & \\
\hline 2 & $65(65.0)$ & - & 65 (34.9) & \\
\hline 3 & $21(21.0)$ & - & $21(11.3)$ & \\
\hline 4 & $6(6.0)$ & - & $6(3.2)$ & \\
\hline 5 & $3(3.0)$ & - & $3(1.6)$ & \\
\hline Location & & & & 0.035 \\
\hline ICA & $34(34.0)$ & 41 (47.7) & 75 (40.3) & \\
\hline$A C A$ & 45 (45.0) & $21(24.4)$ & $66(35.5)$ & \\
\hline MCA & $9(9.0)$ & $10(11.6)$ & $19(10.2)$ & \\
\hline Post cir & $12(12.0)$ & $14(16.3)$ & $26(14.0)$ & \\
\hline Size of aneurysm (mm) & $7.1 \pm 3.7$ & $6.4 \pm 4.0$ & $6.8 \pm 3.9$ & 0.224 \\
\hline Aspect ratio & $2.0 \pm 1.1$ & $1.5 \pm 1.0$ & $1.8 \pm 1.1$ & 0.001 \\
\hline Side of approach & & & & 0.653 \\
\hline Right & $38(38.0)$ & 36 (41.9) & 74 (39.8) & \\
\hline Left & $62(62.0)$ & $50(58.1)$ & $112(60.2)$ & \\
\hline Coiling technique & & & & 0.000 \\
\hline Simple & $70(70.0)$ & $26(30.2)$ & $96(51.6)$ & \\
\hline BAC & $22(22.0)$ & 17 (19.8) & $39(21.0)$ & \\
\hline SAC & $8(8.0)$ & $43(50.0)$ & $51(27.4)$ & \\
\hline Raymond class & & & & 0.035 \\
\hline 1 & $68(68.0)$ & 47 (54.7) & $115(61.8)$ & \\
\hline ॥ & $23(23.0)$ & $20(23.3)$ & $43(23.1)$ & \\
\hline III & $9(9.0)$ & $19(22.1)$ & $28(15.1)$ & \\
\hline Packing density (\%) & $37.1 \pm 11.8$ & $35.8 \pm 10.5$ & $36.5 \pm 11.2$ & 0.424 \\
\hline Procedure time (min) & $155.2 \pm 71.4$ & $133.8 \pm 59.1$ & $145.3 \pm 66.7$ & 0.029 \\
\hline Presence of DPL & 57 (57.0) & 37 (43.0) & 94 (50.5) & 0.077 \\
\hline No of DPL & $3.2 \pm 5.2$ & $1.8 \pm 3.5$ & $2.6 \pm 4.5$ & 0.025 \\
\hline Permanent PRC & $3(3.0)$ & $1(1.2)$ & $4(2.2)$ & 0.625 \\
\hline PRC & & & & 0.034 \\
\hline No & $3(83.0)$ & $81(94.2)$ & $164(88.2)$ & \\
\hline IPR & $12(12.0)$ & $2(2.3)$ & $14(7.5)$ & \\
\hline TE & $5(5.0)$ & $3(3.5)$ & $8(4.3)$ & \\
\hline Outcome at discharge & & & & 0.043 \\
\hline Good recovery & $90(90.0)$ & 85 (98.8) & $175(94.1)$ & \\
\hline Moderate disability & 7 (7.0) & $1(1.2)$ & $8(4.3)$ & \\
\hline Severe disability & $2(2.0)$ & 0 & $2(1.1)$ & \\
\hline Dead & $1(1.0)$ & 0 & $1(0.5)$ & \\
\hline
\end{tabular}

HHG = Hunt \& Hess grade; ICA = internal carotid artery; $\mathrm{ACA}=$ anterior cerebral artery; $\mathrm{MCA}=$ middle cerebral artery; Post cir $=$ posterior circulation; $\mathrm{BAC}=$ balloon assisted coiling; $S A C=$ stent assisted coiling; $\mathrm{DPL}=$ diffusion positive lesion; $\mathrm{PRC}=$ procedure related complication; IPR = intraprocedural rupture; TE = thromboembolism 
use BAC was made by the attending surgeon on a case-by-case basis. All BAC procedures were performed using Hyperform or Hyperglide balloons (eV3, Irvine, CA, United States). Embolization was deemed sufficient when dense coil packing was obtained and no additional coil could be safely inserted. Aneurysm selection was considered difficult if placement of the microcatheter into the aneurysmal sac after introduction of the microcatheter took $>20$ minutes, or aneurysm superselection with microcatheter required $\geq 3$ times microcatheter reshaping.

After the coil embolization, the patients routinely underwent DWI within 24 hours post-procedure as a protocol for detection of procedure-related ischemic complications. Imaging was performed on either a 1.5 T or 3T MR imaging system (Phillips, Amsterdam, Netherland). Any area of restricted diffusion, regardless of size or clinical symptoms was considered a positive result.

\section{Statistical analysis}

Statistical analyses were performed using SPSS 18.0 for Windows (SPSS Inc. Chicago, IL, United States). Continuous variables were expressed as mean \pm SD. The significance of differences between two groups was examined using independent sample $t$-test for continuous variables, and two-way Fisher's exact test for categorical variables. The significance of differences among the three groups was examined using one way ANOVA for continuous variables. Logistic regression analysis was used for identification of the risk factors for DPLs. A $p$ value $<0.05$ was considered statistically significant.

\section{RESULTS}

Sixty five of the 100 patients with ruptured aneurysms were women. Anterior cerebral artery (ACA) aneurysms were most common followed by internal carotid artery (ICA) aneurysms, posterior circulation aneurysms and middle cerebral artery (MCA) aneurysms. The techniques used for coiling were BAC in 22, SAC in eight, and simple coiling alone in 70. Mean packing density was $37.1 \%$. Complete obliteration of the aneurysm was achieved in $68 \%$, neck remnant in $23 \%$ and aneurysm sac filling in $8 \%$.

Seventy one of 86 unruptured aneurysms were in women. The location of aneurysms was ICA in 41, ACA in 21, posterior circulation in 14, and MCA in 10. The techniques used for coiling were BAC in 17, SAC in 43, and simple coiling in 26. Mean packing density was $35.8 \%$. Complete obliteration of the aneurysm was achieved in 54.7\%, neck remnant in $23.3 \%$ and aneurysm sac filling in $22.1 \%$.

Even though there were 14 intra-procedural ruptures and 8 intra-procedural TE complications, the permanent complication rate was only $2.1 \%(4 / 186)$ (Table 1).

Overall, DPLs were observed in 50.5\% (94/186). DPLs had a tendency to increase in ruptured aneurysms compared with unruptured aneurysms (57\% vs. $43 \%, p=0.077$ ). The mean number of DPLs was significantly higher in the ruptured group (3.22 \pm 5.22 , range: 0 -30) than in the unruptured group (1.77 \pm 3.46, range: $0-16)(p=0.025)$ (Table 1$)$. Increasing age influenced the occurrence of DPLs in patients with ruptured aneurysms, however, the difference did not reach statistical significance $(p=0.056)$ (Table 2). ACA aneurysms showed the highest incidence of DPLs, followed by ICA aneurysms, but the difference was not statistically significant $(p>0.05)$. Endovascular neurosurgeon's experience did not affect the occurrence of DPLs in both groups even though the initial period (before year 2010) showed high incidence of DPLs (Table 2).

Aneurysm selection was considered difficult in $21.0 \%$. However, the occurrence of DPLs was not dependent on the difficulty of aneurysm selection. Aneurysm size, neck size and aspect ratio did not influence the occurrence of DPLs.

The left side approach showed more DPL in all anterior circulation aneurysms $(n=160)$, however, the difference was not statistically significant $(p>0.05)$ (Table 2). Based on the coiling technique, DPLs oc- 
Table 2. Incidence of diffusion positive lesions according to the clinical characteristics in patients with ruptured and unruptured aneurysms

\begin{tabular}{|c|c|c|c|c|c|c|}
\hline \multirow{2}{*}{ Variables } & \multicolumn{3}{|c|}{ Ruptured group } & \multicolumn{3}{|c|}{ Unruptured group } \\
\hline & DWI $1+1$ & DWI H & $\rho$ value & DWI $1+1$ & DWI (-) & $\rho$ value \\
\hline No & $57(57)$ & $43(43)$ & & $37(43)$ & $49(57)$ & \\
\hline Age & $56.7 \pm 11.3$ & $51.7 \pm 14.8$ & 0.056 & $60.7 \pm 12.5$ & $58.9 \pm 13.3$ & 0.520 \\
\hline Female sex & $40(70.2)$ & $25(58.1)$ & 0.290 & 33 (89.2) & 38 (77.6) & 0.251 \\
\hline Location & & & 0.556 & & & 0.694 \\
\hline ICA & 20 (58.8) & 14 (41.2) & & 17 (41.5) & 24 (58.5) & \\
\hline$A C A$ & $28(62.2)$ & 17 (37.8) & & 11 (52.4) & $10(47.6)$ & \\
\hline MCA & $4(44.4)$ & $5(55.6)$ & & $3(30)$ & 7 (70) & \\
\hline Post cir & $5(41.7)$ & 7 (58.3) & & $6(42.9)$ & $8(57.1)$ & \\
\hline Surgeon's experience & & & 0.210 & & & 0.330 \\
\hline$<2010$ & $16(72.7)$ & $6(27.3)$ & & $8(50.0)$ & $8(50.0)$ & \\
\hline $2010-2011$ & $22(56.4)$ & 17 (43.6) & & $6(28.6)$ & 15 (71.4) & \\
\hline $2012-2013$ & 19 (48.7) & 20 (51.3) & & $23(46.9)$ & $26(53.1)$ & \\
\hline Aneurysm selection & & & 1.000 & & & 1.000 \\
\hline Easy & $44(56.4)$ & 34 (43.6) & & $29(42.6)$ & 39 (57.4) & \\
\hline Difficult & 13 (59.1) & 9 (40.9) & & $8(44.4)$ & $10(55.6)$ & \\
\hline Aneurysm size $(\mathrm{mm})$ & & & 0.681 & & & 0.629 \\
\hline$\leq 7$ & 36 (59.0) & $25(41.0)$ & & $28(45.2)$ & 34 (54.8) & \\
\hline$>7$ & $21(53.8)$ & $18(46.2)$ & & $9(37.5)$ & $15(62.5)$ & \\
\hline Neck size(mm) & & & 0.664 & & & 0.185 \\
\hline$\leq 4$ & $38(55.1)$ & 31 (44.9) & & $25(50.0)$ & $25(50.0)$ & \\
\hline$>4$ & 19 (61.3) & $12(38.7)$ & & 12 (33.3) & 24 (66.7) & \\
\hline Aspect ratio & & & 0.482 & & & 0.732 \\
\hline$\leq 1$ & $2(33.3)$ & $4(66.7)$ & & 12 (38.7) & $19(61.3)$ & \\
\hline $1-1.5$ & $13(54.2)$ & $11(45.8)$ & & 10 (43.5) & 13 (56.5) & \\
\hline$>1.5$ & 42 (60.0) & 28 (40.0) & & 15 (48.4) & 16 (51.6) & \\
\hline Approach side $(n=170)$ & & & 0.674 & & & 0.040 \\
\hline Right & 21 (55.3) & 17 (44.7) & & 10 (29.4) & $24(70.6)$ & \\
\hline Left & $33(60.0)$ & $22(40.0)$ & & 23 (53.5) & 20 (46.5) & \\
\hline Coiling technique & & & 0.713 & & & 0.084 \\
\hline Simple & 38 (54.3) & 32 (45.7) & & 15 (57.7) & 11 (42.3) & \\
\hline BAC & $14(63.6)$ & $8(36.4)$ & & $4(23.5)$ & $13(76.5)$ & \\
\hline SAC & $5(62.5)$ & $3(37.5)$ & & 18 (41.9) & $25(58.1)$ & \\
\hline Packing density & $37.2 \pm 13.3$ & $37.0 \pm 9.6$ & 0.928 & $34.6 \pm 9.7$ & $36.6 \pm 11.1$ & 0.401 \\
\hline Detach method & & & 0.228 & & & 0.511 \\
\hline Mechanical (n=99) & 30 (63.8) & $17(36.2)$ & & $24(46.2)$ & 28 (53.8) & \\
\hline Electrical, or combined $(n=87)$ & 27 (50.9) & $26(49.1)$ & & $13(38.2)$ & $21(61.8)$ & \\
\hline
\end{tabular}

$\mathrm{DWI}=$ diffusion weighted image; ICA = internal carotid artery; $\mathrm{ACA}=$ anterior cerebral artery; $\mathrm{MCA}=$ middle cerebral artery; Post cir = posterior circulation; $\mathrm{BAC}=$ balloon assisted coiling; $\mathrm{SAC}=$ stent assisted coiling

curred in $23.5 \%$ of $\mathrm{BAC}, 41.9 \%$ of SAC and $57.7 \%$ of simple coiling in unruptured aneurysms $(p=0.08)$. However, in patients with ruptured aneurysms, occurrence of DPLs did not differ among simple coiling
$(54.3 \%), \operatorname{SAC}(62.5 \%)$, and $\mathrm{BAC}(63.6 \%)$ groups $(p=$ 0.71) (Table 2).

Mean number of DPLs was the highest in the SAC group (5.25), followed by BAC (3.41) and simple coil- 


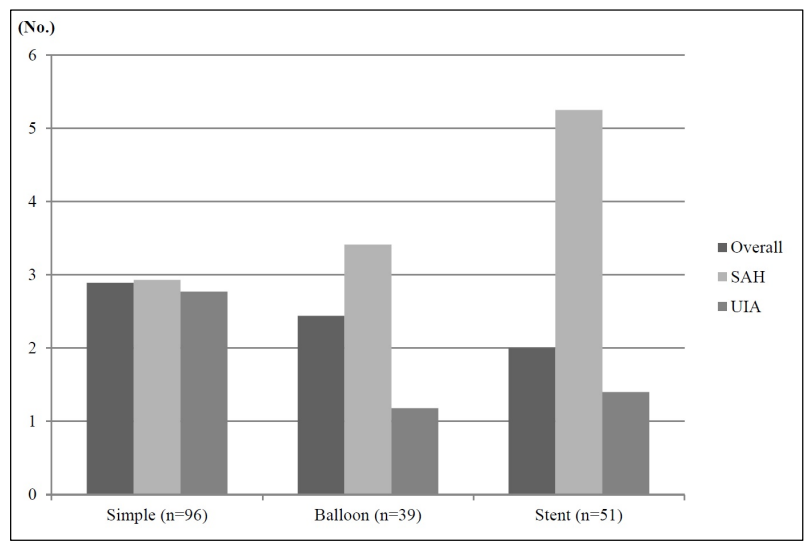

Fig. 2. Bar graphs showing the mean number of diffusion positive lesions (DPLS) according to the treatment modalities. Mean number of DPL was the highest in stent assisted coiling followed by balloon assisted coiling $(\rho>0.05)$ in the subarachnoid hemorrhage (SAH) group; however, in the unruptured group, balloon assisted coiling showed the lowest mean number of DPLs even though the difference was not statistically significant.

ing group (2.93) in ruptured aneurysms. However, the BAC group showed the lowest mean number of DPLs (1.18) in unruptured aneurysms (Fig. 2). In comparison of simple coiling and BAC in unruptured aneurysm patients, the occurrence of DPLs was significantly lower in BAC $(p=0.034)$, however, there was no difference in ruptured aneurysm patients $(p=$ 0.472) (Fig. 3).

Detachment method did not influence the occurrence of DPL in both groups. Mean packing density was not different between the diffusion positive and diffusion negative groups (Table 2).

Logistic regression analysis revealed that age $>55$ years was the only independent risk factor for the occurrence of DPLs in the entire patient cohort on ad-

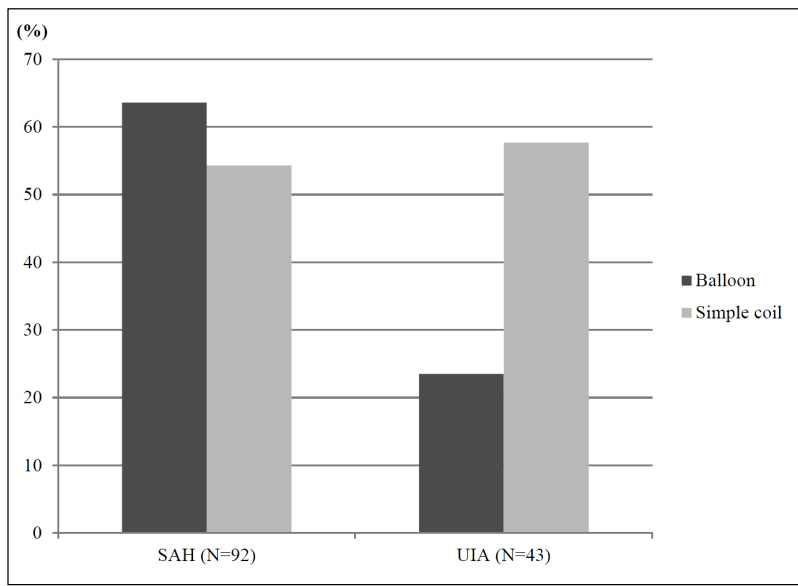

Fig. 3. Bar graphs showing the incidence of DPLs. Balloon assisted coiling significantly lowers the incidence of DPLs in unruptured intracranial aneurysm (UIA) patients $(\rho=0.034)$, however, this effect was absent in SAH patients $(p=0.472)$.

justing rupture status, side of approach, selection difficulty and coiling methods (Table 3).

\section{DISCUSSION}

Occurrence of DPLs after coil embolization of cerebral aneurysm has been reported in $10-61 \%$ of the cases. ${ }^{24) 12-14) 17) 19) 20) 23-25) 27) ~ D P L s ~ c a n ~ b e ~ p r o d u c e d ~ b y ~ m a-~}$ nipulation of the guiding catheter, difficulty of aneurysm selection, event during framing coil, amount of coils, detachment method, surgeon's experience and perioperative antithrombotic agent. ${ }^{613) 14) 17) 23) 25)}$ We investigated the occurrence of TE based on the DPLs on postoperative DWI, in ruptured and unruptured aneurysms treated with coil embolizations. The overall occurrence of DPLs was $50.5 \%$ in our study. The

Table 3. Logistic regression analysis for the risk factors of diffusion positive lesions in 186 patients

\begin{tabular}{|c|c|c|c|}
\hline Parameter & Odd ratio & $95 \% \mathrm{Cl}$ & $\rho$ value \\
\hline Age > 55 years & 1.95 & $1.041-3.661$ & 0.037 \\
\hline $\mathrm{SAH}$ & 1.85 & $0.927-3.681$ & 0.081 \\
\hline Left side approach & 1.35 & $0.731-2.480$ & 0.339 \\
\hline Selection difficulty & 1.302 & $0.623-2.718$ & 0.482 \\
\hline Coil method (simple) & 1 & & 0.626 \\
\hline Balloon assisted & 1.237 & $0.554-2.763$ & 0.604 \\
\hline Stent assisted & 0.845 & $0.339-2.107$ & 0.717 \\
\hline
\end{tabular}


number of DPLs in patients with ruptured aneurysms was higher than that of unruptured aneurysms. The reason that DPLs occurred more frequently in patients with ruptured aneurysm was related to the hypercoagulable state in SAH conditions. ${ }^{3)}$ When the aneurysm ruptures, activation of the blood coagulation system, as well as the fibrinolytic system (especially the secondary fibrinolysis) occurs early in the course of $\mathrm{SAH}$, particularly in patients with a severe neurological condition. ${ }^{79)}$

Another reason might be related to different protocols of antithrombotic medication and timing. Dual antiplatelet agents were not administered in most ruptured aneurysm patients, except for SAC. In addition, intravenous heparin was not injected until framing coil packing in ruptured aneurysms, however, it was administered immediately after placement of the femoral sheath in unruptured aneurysms.

Hwang et al. ${ }^{5)}$ reported that antiplatelet premedication lowered the periprocedural thromboembolic complication rate in unruptured aneurysms treated using the multiple microcatheter technique and did not increase the risk of hemorrhage.

In our study, increasing age influenced the occurrence of DPLs and age of $>55$ years predicted an increased risk of DPLs. Increasing age might be related to the tortuous large vessels including aortic arch, brachiocephalic trunk, common and internal carotid arteries, and tortuous origin of the vertebral artery. Catheterizing tortuous arteries could increase the risk of TE. Chung et al. ${ }^{9)}$ reported that the occurrence of DPL increased at an older age of $>60$ years. The authors assumed that more atherosclerotic changes in the cerebral artery were present in older patients, and that these patients were more vulnerable to TE events in aneurysm-unrelated territories as a result of routine procedures, such as the use of a guiding catheter.

Murayama et al. ${ }^{13)}$ reported that risk of TE was expected to increase in the hands of inexprienced clinicians. However, in this study, endovascular neurosurgeon's experience did not affect the occurrence of DPLs in both groups even though DPLs tended to occur more frequently in the initial period before 2010.

ACA aneurysms had the highest incidence of DPL in our study. We thought that the possible reasons why the ACA aneurysm had a high incidence of DPL might be related to the acute angle of ACA origin from ICA bifurcation and relatively small diameter of A1, A2 and anterior communicating arteries. Increase in procedure time or a significant drop in systolic BP under general anesthesia may have increased the risk of TE, particularly in this specific location.

Sim et al. ${ }^{22)}$ reported a high incidence of DPLs in the left side approach as compared to the right side approach, since catheterization of the left common carotid artery was more difficult than that of the right, due to the acute angle of the left common carotid artery from the aortic arch. In our study, we also demonstrated similar results in patients with unruptured aneurysms.

The role of the balloon in the occurrence of DPL is controversial. ${ }^{810-12) 1415)}$ Many authors have postulated that the use of a balloon during coiling might increase the occurrence of ischemic lesion after embolization of cerebral aneurysms. ${ }^{1114) 24)}$ Nelson et al. ${ }^{14)}$ postulated that the increased number of guidewires and microcatheters necessary for BAC added complexity to the technique, which might increase the risk of complication. Malek et al. ${ }^{11)}$ reported that the higher rate of TE complications was the result of placement of an additional balloon microcatheter in the parent artery. Soeda et al. ${ }^{24)}$ reported that the balloon technique required sophisticated handling, frequent use of guidewires and microcatheters, the need for temporary occlusion of the parent vessel, and repeated inflation and deflation of the balloon, which might have increased occurrence of TE event.

However, contradictory results of equal or decreased incidence of ischemic lesion after BAC, have been reported by some authors. ${ }^{16(8)(12) 18 / 19921)}$ In this study, occurrence of DPL was significantly lower in 
the BAC group compared with simple coiling group in unruptured aneurysms only.

The exact reason why the BAC in patients with unruptured aneurysms showed less DPLs is not clear. Possible reasons might be related to premedication with dual antiplatelet agents." In our institution, scheduled unruptured aneurysm embolization was performed on dual antiplatelet premedication. After the embolization procedure, dual antiplatelet medication was not maintained in simple or BAC cases, but only in cases of SAC. However, in ruptured aneurysms, BAC was performed without antiplatelet medication. This difference might have influenced the occurrence of DPLs. Another difference was the timing of intravenous heparin injection. In the unruptured group, heparin was injected intravenously early in the procedure whereas in the ruptured group, it was injected after framing coil packing or just before balloon inflation.

Some studies have reported an embolic complication by electrolytic coil detachment. ${ }^{16) 19) 26)}$ Padolecchia et al. ${ }^{16)}$ observed that electrothrombosis might have a negative effect, in that it could promote unwanted thrombus formation, with possible consequent downstream thromboembolic complications. In our study, no difference was observed between electrolytic and mechanical coil detachment likely due to prevention of thrombus formation by appropriate intra-procedural anticoagulant medication. We rarely used electrolytic detachment coils alone. Most of the electrolytic detachment group included combined mechanical detachment and electrolytic detachment coils.

Despite the frequent occurrence of DPLs following coil embolization of cerebral aneurysms, most of the DPLs were asymptomatic. The incidence of symptomatic TE events was reportedly 5-28\%. ${ }^{15) 17) 19) 23)}$ Similarly in our study, the incidence of DPLs was 50.5\%, however, TE event occurred in $4.3 \%$ of cases. The permanent complication resulting from TE was only $2.2 \%$. This discrepancy could be explained by the fact that a relatively small part of the brain was affected, and DPL mostly tended to localize in the silent cortex in- stead of the eloquent area. ${ }^{19)}$

Gentle manipulation of cerebral or extracerebral arteries using various endovascular devices is mandatory in order to reduce the occurrence of DPL after coil embolization. In addition, preoperative antiplatelet medication might be helpful if possible.

The limitation of this study included its retrospective design, which excluded 87 cases in whom performance of postoperative DWI was impossible due to poor clinical conditions. Exclusion of patients in poor clinical condition, may have led to underestimation of overall permanent complication and symptomatic TE event rate, because intraprocedural complication can itself lead to poor condition. Another limitation of this study was the different preoperative antiplatelet therapy and different timing of intravenous anticoagulant injection between ruptured and unruptured groups.

\section{CONCLUSION}

DPLs occurred more frequently in ruptured aneurysms and at an older age. Even though most DPLs are asymptomatic careful manipulation of cerebral or extracerebral arteries using various endovascular devices is important in order to reduce the occurrence of DPLs, particularly in patients $>55$ years old. BAC appeared to reduce TE events in patient with unruptured aneurysms. However, conduct of further study with a prospective design is required in order to confirm these results.

\section{REFERENCES}

1. Albayram S, Selcuk H, Kara B, Bozdag E, Uzma O, Kocer $\mathrm{N}$, et al. Thromboembolic events associated with balloon-assisted coil embolization: evaluation with diffusion-weighted MR imaging. AJNR Am J Neuroradiol. 2004 Nov-Dec;25(10):1768-77.

2. Altay T, Kang HI, Woo HH, Masaryk TJ, Rasmussen PA, Fiorella DJ, et al. Thromboembolic events associated with endovascular treatment of cerebral aneurysms. J Neurointerv Surg. 2011 Jun;3(2):147-50.

3. Ettinger MG. Coagulation abnormalities in subarachnoid 
hemorrhage. Stroke. 1970 May-Jun;1(3):139-42.

4. Fujita K, Tamaki N. [Coagulation abnormalities in stroke-disseminated intravascular coagulation as a complication of subarachnoid hemorrhage]. Rinsho Byori. 1991 Jul;39(7):729-35. Japanese.

5. Hwang G, Jung C, Park SQ, Kang HS, Lee SH, Oh CW, et al. Thromboembolic complications of elective coil embolization of unruptured aneurysms: the effect of oral antiplatelet preparation on periprocedural thromboembolic complication. Neurosurgery. 2010 Sep;67(3):743-8; discussion 748 .

6. Ilveskero S, Juvela S, Siironen J, Lassila R. D-dimer predicts outcome after aneurysmal subarachnoid hemorrhage: no effect of thromboprophylaxis on coagulation activity. Neurosurgery. 2005 Jul;57(1):16-24; discussion 16-24.

7. Ji Y, Meng QH, Wang ZG. Changes in the coagulation and fibrinolytic system of patients with subarachnoid hemorrhage. Neurol Med Chir (Tokyo). 2014 Jun 17;54(6) :457-64.

8. Jo KI, Yeon JY, Kim KH, Jeon P, Kim JS, Hong SC. Predictors of thromboembolism during coil embolization in patients with unruptured intracranial aneurysm. Acta Neurochir (Wien). 2013 Jun;155(6):1101-6.

9. Kusanagi H, Teramoto A, Shimura T. [The relationship between the severity of a neurological condition and the blood coagulation/fibrinolytic system in patients with spontaneous subarachnoid hemorrhage]. No Shinkei Geka. 2002 Apr;30(4):399-403. Japanese.

10. Lefkowitz MA, Gobin YP, Akiba Y, Duckwiler GR, Murayama Y, Guglielmi G, et al. Balloon-assisted Guglielmi detachable coiling of wide-necked aneurysms: Part II-clinical results. Neurosurgery. 1999 Sep;45(3):531-7; discussion 537-8.

11. Malek AM, Halbach VV, Phatouros CC, Lempert TE, Meyers PM, Dowd CF, et al. Balloon-assist technique for endovascular coil embolization of geometrically difficult intracranial aneurysms. Neurosurgery. 2000 Jun;46(6):1397-406; discussion 1406-7.

12. Moret J, Cognard C, Weill A, Castaings L, Rey A. The "remodelling technique" in the treatment of wide neck intracranial aneurysms. Angiographic results and clinical follow-up in 56 cases. Interv Neuroradiol. 1997 Mar;3(1):21-35.

13. Murayama Y, Vinuela F, Duckwiler GR, Gobin YP, Guglielmi G. Embolization of incidental cerebral aneurysms by using the Guglielmi detachable coil system. J Neurosurg. 1999 Feb;90(2):207-14.

14. Nelson PK, Levy DI. Balloon-assisted coil embolization of wide-necked aneurysms of the internal carotid artery: medium-term angiographic and clinical follow-up in 22 patients. AJNR Am J Neuroradiol. 2001 Jan;22(1):19-26.

15. Nina P, Schisano G, Chiappetta F, Luisa Papa M, Maddaloni E, Brunori A, et al. A study of blood coagulation and fibrinolytic system in spontaneous subarachnoid hemorrhage. Correlation with hunt-hess grade and outcome. Surg Neurol. 2001 Apr;55(4):197-203.

16. Padolecchia R, Guglielmi G, Puglioli M, Castagna M, Nardini V, Collavoli PL, et al. Role of electrothrombosis in aneurysm treatment with Guglielmi detachable coils: an in vitro scanning electron microscopic study. AJNR Am J Neuroradiol. 2001 Oct;22(9):1757-60.

17. Pelz DM, Lownie SP, Fox AJ. Thromboembolic events associated with the treatment of cerebral aneurysms with Guglielmi detachable coils. AJNR Am J Neuroradiol. 1998 Sep;19(8):1541-7.

18. Pierot L, Spelle L, Vitry F; ATENA Investigators. Immediate clinical outcome of patients harboring unruptured intracranial aneurysms treated by endovascular approach: results of the ATENA study. Stroke. 2008 Sep;39(9):2497-504.

19. Rordorf G, Bellon RJ, Budzik RE Jr, Farkas J, Reinking GF, Pergolizzi RS, et al. Silent thromboembolic events associated with the treatment of unruptured cerebral aneurysms by use of Guglielmi detachable coils: prospective study applying diffusion-weighted imaging. AJNR Am J Neuroradiol. 2001 Jan;22(1):5-10.

20. Ruigrok YM, Slooter AJ, Rinkel GJ, Wijmenga C, Rosendaal FR. Genes influencing coagulation and the risk of aneurysmal subarachnoid hemorrhage, and subsequent complications of secondary cerebral ischemia and rebleeding. Acta Neurochir (Wien). 2010 Feb;152(2):257-62.

21. Shapiro M, Babb J, Becske T, Nelson PK. Safety and efficacy of adjunctive balloon remodeling during endovascular treatment of intracranial aneurysms: a literature review. AJNR Am J Neuroradiol. 2008 Oct;29(9):1777-81.

22. Sim SY, Shin YS. Silent microembolism on diffusion-weighted MRI after coil embolization of cerebral aneurysms. Neurointervention. 2012 Sep;7(2):77-84.

23. Soeda A, Sakai N, Murao K, Sakai H, Ihara K, Yamada $\mathrm{N}$, et al. Thromboembolic events associated with Guglielmi detachable coil embolization with use of diffusionweighted MR imaging. Part II. Detection of the microemboli proximal to cerebral aneurysm. AJNR Am J Neuroradiol. 2003 Nov-Dec;24(10):2035-8.

24. Soeda A, Sakai N, Sakai H, Iihara K, Yamada N, Imakita S, et al. Thromboembolic events associated with Guglielmi detachable coil embolization of asymptomatic cerebral aneurysms: evaluation of 66 consecutive cases with use of diffusion-weighted MR imaging. AJNR Am J Neuroradiol. 2003 Jan;24(1):127-32.

25. Spiotta AM, Bhalla T, Hussain MS, Sivapatham T, Batra A, Hui $F$, et al. An analysis of inflation times during balloon-assisted aneurysm coil embolization and ischemic complications. Stroke. 2011 Apr;42(4):1051-5.

26. Touho H, Hino A, Suzuki K, Kubo T, Hirakawa K. [Coagulation-fibrinolysis abnormalities in acute stage of subarachnoid hemorrhage (Part 1)- with special reference to the relation between cerebral vasospasm and fibrinopeptides A and B beta]. No To Shinkei. 1984 Oct;36(10):1009-14. Japanese.

27. Vinuela F, Duckwiler G, Mawad M. Guglielmi detachable coil embolization of acute intracranial aneurysm: perioperative anatomical and clinical outcome in 403 patients. J Neurosurg. 1997 Mar;86(3):475-82. 